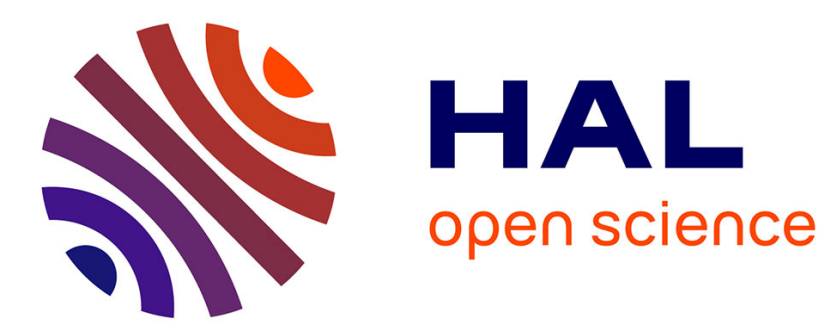

\title{
Le mythe de la cuisine viennoise \\ Catherine Horel
}

\section{- To cite this version:}

Catherine Horel. Le mythe de la cuisine viennoise. Bulletin de l'Institut Pierre Renouvin, 2020. halshs-03090248

\section{HAL Id: halshs-03090248 https://shs.hal.science/halshs-03090248}

Submitted on 29 Dec 2020

HAL is a multi-disciplinary open access archive for the deposit and dissemination of scientific research documents, whether they are published or not. The documents may come from teaching and research institutions in France or abroad, or from public or private research centers.
L'archive ouverte pluridisciplinaire HAL, est destinée au dépôt et à la diffusion de documents scientifiques de niveau recherche, publiés ou non, émanant des établissements d'enseignement et de recherche français ou étrangers, des laboratoires publics ou privés. 


\section{Résumé}

Les produits alimentaires et la gastronomie ont circulé dans l'Empire, constituant un lieu de mémoire de la cuisine habsbourgeoise. Les fonctionnaires, militaires, enseignants ont vu leurs goûts changer au gré de leurs mutations : leurs femmes ne devaient pas seulement s'adapter à la langue de leur cuisinière, mais aussi à ses pratiques et aux ingrédients du marché local. Les livres de cuisine à leur disposition concentraient la diversité de la cuisine de la monarchie. Le vocabulaire reflète ces transferts au travers de mots qui sont des créations transnationales.

Mots-clés : Empire des Habsbourg - Cuisine viennoise - Livres de cuisine Héritage culturel - Invention de la tradition.

\section{Abstract}

\section{The Myth of the Viennese Cuisine}

Food and gastronomy travelled from one end of the Empire to the other, forming the cultural heritage of Habsburg cuisine. Civil servants, officers, academics came back from their various postings with a changed taste: their wives had to adapt not only to the foreign language spoken by the cook but also to her cuisine as well as the ingredients offered in the market place. Books which were written for them that gathered all the diversity of 'Habsburg' cuisine. The vocabulary bears witness to these transfers: many words for ingredients, vegetables, are typical 'Habsburg' constructions.

Keywords: Habsburg Empire - Viennese cooking - Cookbooks - Cultural heritage - Invention of tradition.

Par son caractère multinational, l'empire des Habsbourg incarne l'hybridité des identités multiples (ethniques, linguistiques, confessionnelles), fruit de la mobilité et des échanges ${ }^{212}$. Au travers de ses divers territoires, un phénomène de traduction et d'intégration des traditions culinaires s'observe du centre vers les périphéries. L'Empire n'a pas seulement adapté et adopté des recettes ottomanes et italiennes, mais celles-ci sont devenues des symboles de sa « Gemütlichkeit » (douceur de

Le présent article est un résumé de "Francis Joseph'sTafe/spitz. The Austro-Hungarian Cooking as an Imperial Project », in llaria Porciani (ed.), Food as Heritage and Nationalism in Europe. Tastes of Diversity, London, Routledge, 2019. 
vivre) : le café dans toutes ses variations possibles, les Strudel (sucré et salé) et les Fisolen (haricots), de même que les vins produits dans de nombreuses régions de la monarchie. Les denrées alimentaires et les pratiques gastronomiques ont circulé d'un bout à l'autre de l'Empire. À partir du milieu du XIX ${ }^{e}$ siècle, l'augmentation et l'accélération de la mobilité a permis non seulement de transporter la nourriture mais elle a aussi donné aux gens la possibilité de voyager pour le travail, les études ou les loisirs, vers des lieux où ils sont entrés en contact avec des pratiques culinaires et des produits inconnus. Les domestiques tchèques venant s'employer à Vienne ont fait de la cuisine de la Bohême une caractéristique de la gastronomie de la capitale. Le développement des chemins de fer s'est traduit par la construction de gares démonstratives où le restaurant est devenu un lieu de transfert culturel des recettes de tous les territoires: c'est ainsi que la soupe hongroise gulyás s'est exportée vers l'ouest où elle a été transformée en ragoût de bœuf. Les fonctionnaires, officiers et enseignants voyaient leurs goûts changer au gré de leurs mutations. Leurs épouses devaient, d'une part, s'adapter à la langue de leur cuisinière, d'autre part, à ses pratiques culinaires et aux ingrédients disponibles sur le marché local. Le vocabulaire témoigne de ces transferts: de nombreux mots sont des créations empruntées aux lexiques slaves, italien, hongrois ; acclimatées à Vienne, elles sont inconnues en allemand.

Grâce à l'union douanière et à la monnaie unique, les produits circulent sans restriction à travers toute la monarchie, popularisés par la publicité que les journaux traduisent dans la langue locale, créant ainsi des références culinaires communes. On trouve des réclames pour les produits de la maison Meinl dans toutes les langues et des succursales de la célèbre épicerie viennoise ouvrent dans les grandes villes de l'Empire. II en résulte que beaucoup de recettes sont absorbées dans ce qui va devenir la cuisine "viennoise", et que des influences sont adaptées ou importées, dont certaines datent $d u x l^{e}$ siècle. Vienne devient un creuset dans le premier sens du terme, où des contributions étrangères (France, Empire ottoman) et locales (pays alpins, Bohême, Hongrie, provinces italiennes) se mélangent. Un patchwork culinaire formé de tous les territoires de l'Empire se constitue et ses plats sont proposés dans les restaurants (Beis/n) de la capitale ${ }^{213}$. Ce

\section{3}

Susanne Breuss, "Zur Bedeutung des kulinarischen für die Konstruktion österreichischer Identität », in Hannes Stekl, Elena Mannová (hrsg.), Heroen, 
processus

légendes et est caractérisé par l'hybridité et la transformation. Des des mythes sont inventés pour expliquer ces

transferts

- dont un des plus célèbres est la Wiener Schnitzel - qui permettent de parler ici d'une véritable "invention de la tradition " pour décrire ce palimpseste qu'est la cuisine viennoise. Des origines géographiques différentes sont associées à des recettes identiques, alors qu'à l'inverse, des termes similaires mais grossièrement traduits passent d'un lexique à l'autre (allemand, slave, latin-Italien, turc, yiddish) causant par la suite d'infinies querelles nationalistes sur l'appartenance d'un plat prétendument " typique ». Dans les diverses régions de l'Empire, consommer des mets dont la recette provient de provinces reculées (Bucovine, Dalmatie) s'apparente à une forme d'exotisme domestique (Binnenexotik). Après la dissolution de l'Empire austro-hongrois, certaines recettes ont acquis le statut de lieu de mémoire, faisant ainsi de la cuisine viennoise le témoignage de la multiplicité des goûts d'un empire où chaque peuple contribuait à son exceptionnelle diversité. Mais que reste-t-il de ces transferts en dehors de Vienne? Ce creuset s'est-il exporté ? En effet, alors que toutes les recettes de l'Empire sont disponibles dans sa capitale, pourquoi ont-elles migré à leur tour vers d'autres provinces ? Pouvait-on déguster de la cuisine tchèque à Sarajevo, ou trouver du paprika de Hongrie à Trieste ? À cet égard, la différence semble résider davantage entre les environnements urbains et ruraux: les paysannes de Galicie, qu'elles soient ruthènes ou polonaises, ont continué à cuisiner comme leurs mères. La créativité, la fantaisie et la curiosité, mais aussi l'adaptabilité étaient des attributs urbains, encouragés par la disponibilité d'une plus grande variété de produits.

La cuisine a joué un rôle non négligeable dans la formation des identités nationales par l'appropriation et la différenciation. Les hôteliers et les cuisiniers ont été des acteurs essentiels de ce processus de transfert, certains écrivant même leurs propres livres de cuisine ou de pâtisserie. Les migrants, généralement originaires des zones rurales, apportaient avec eux leurs traditions culinaires mais devaient en même temps s'adapter à la disponibilité et au prix des ingrédients, ce qui nécessitait souvent de modifier les recettes voire de les remplacer en assimilant celles appartenant à leur nouvel environnement. Ils inspiraient en retour les

Mythen, Identitäten, die Slowakei und Österreich im Vergleich, Vienne, WUV, 2003, p. 351-372. 
pratiques de leurs voisins ou les indisposaient par l'odeur de certaines préparations. Dans les cours des immeubles de Vienne, Prague et Budapest, les cuisines donnaient sur les coursives extérieures, les Pawlatschen, de sorte que tous pouvaient entendre, sentir et parfois goûter, ce qui mijotait chez le voisin.

Ces transferts sont un phénomène urbain, pratiquement inconnu dans les villages où les gens maintenaient leurs traditions culinaires et n'achetaient que fort rarement des produits "exotiques" au marché de ville la plus proche, en premier lieu en raison de leur coût souvent élevé. Toutefois, l'urbanisation attirait de plus en plus de paysans vers les cités de toutes tailles : du centre régional voisin jusque vers les métropoles de Vienne et Budapest. Dans les villes moyennes, le commerce se développe considérablement, permettant à de nombreuses boutiques de vendre des denrées dont la provenance va bien au-delà de la campagne environnante. Dans les villes plus grandes, la principale raison de l'évolution des habitudes de consommation était le chemin de fer. Les distances vers la capitale régionale se réduisirent et permirent par conséquent aussi le voyage en direction des grandes villes ${ }^{214}$. Le restaurant de la gare était l'endroit privilégié où l'on pouvait se voir proposer de la cuisine dite viennoise. Considéré comme une porte vers le monde extérieur, il devait offrir à la fois des plats appartenant au patrimoine local et procurer également une touche exotique inspirée de la capitale. L'instauration du service militaire obligatoire fit voyager de jeunes campagnards à l'autre bout de l'Empire. Les cuisiniers de l'armée apportèrent selon leurs origines leur touche personnelle à cette cuisine habsbourgeoise.

Durant les dernières décennies $d u \mathrm{XIX}^{\mathrm{e}}$ siècle les villes de la monarchie virent s'ouvrir un nombre considérable de café-restaurants. Ils prenaient souvent des noms éloquents, diffusant le patriotisme dynastique et les références à Vienne. Au-delà de ces appellations ils étaient des relais de la culture impériale de l'Autriche-Hongrie. Les habitants des localités les plus éloignées avaient ainsi l'impression que la capitale s'était rapprochée d'eux. La variété du menu était à l'aune du choix des journaux disponibles dans le café, preuve de son ouverture sur le monde et gage d'excellence

\section{4}

Voir l'histoire humoristique "Die Gans von Podwolotschyska », in Roda Roda, Rote Weste und Monokel. Das neue Roda Roda Buch, Munich, DTV, 1999, p. 136-140. 
culturelle. De nouvelles brasseries ouvraient également, parfois dans les banlieues, dans les parcs ainsi que sur les lieux boisés prisés des excursionnistes du dimanche. Même dans les plus modestes tavernes, un nouveau mode de consommation se faisait jour. Ceci était souvent dû au fait que le restaurateur venait d'une autre région ou bien qu'il revenait dans son pays natal après avoir accumulé de l'expérience ailleurs. Dans la plupart des villes hors de l'Autriche à proprement parler, l'allemand demeurait une langue majeure, de sorte qu'un esprit entreprenant de Graz, par exemple, pouvait tenter sa chance à Czernowitz ou à Temesvár où il allait en outre acquérir en quelques années des notions d'une ou plusieurs langues locales. Les changements de propriétaire ou l'ouverture d'un nouveau café-restaurant étaient l'occasion de faire de la publicité par voie de presse, qui mentionnait parfois l'origine et les références du nouveau tenancier. Si l'on annonçait qu'un brasseur tchèque s'installait à Sarajevo, les futurs clients se félicitaient de cette nouvelle car ils supposaient que l'homme s'y connaissait en matière de bière.

Les cuisiniers étaient les truchements vers la diversité des goûts en adaptant et interprétant les recettes apprises ou découvertes durant leurs années de formation et leurs voyages à travers toute la monarchie. Cette profession, au même titre que beaucoup d'autres, était caractérisée par un fort taux de mobilité. En revanche la cuisinière employée dans les villes moyennes venait le plus souvent de la campagne environnante, mais dans les grandes métropoles elle pouvait avoir parcouru une plus longue distance. À Vienne, la plupart des immigrants venaient de Moravie et de Bohême, parmi eux la majorité des femmes devenaient domestiques dans les foyers bourgeois où elles assuraient la cuisine et souvent la garde des enfants $^{215}$. Ce sont elles qui ont donné à la gastronomie viennoise ses traits les plus célèbres ${ }^{216}$ : les Buchteln (une brioche tchèque dénommée originellement buchta), le Powidl (une marmelade de prunes extrêmement dense que les Polonais se sont appropriés sous le terme de powidła) mais que l'on trouve jusqu'en Transylvanie, et enfin les knedlíky (knödel). Les

\section{5}

Voir l'hommage de Franz Werfel à sa nourrisse tchèque, Barbara oder die Frömmigkeit, Francfort, Fischer, 1990.

216

Rolf Schwendter, Armeessen, Reichespeisen: neuere Sozialgeschichte der zentraleuropäischen Gastronomie, Wien, Promedia, 1995. 
pâtes, avec toutes sortes de variantes sous forme de gnocchi, quenelles etc., connaissent toutefois des différences substantielles. En descendant vers le sud, le Knödel devient de la pasta (Slovénie, provinces italiennes, Dalmatie), et vers l'est, il se transforme en petites pâtes destinées à la soupe (galuska en Hongrie), en ravioli (pierogi polonais ou varenyky ukrainiens), ou constitue un plat en soit agrémenté de fromage et d'autres ingrédients (halušky slovaques ou sztrapacska hongroises). Confrontée à ces nouveautés, la maîtresse de maison peut soit essayer d'enseigner à la cuisinière d'autres traditions, parfois les siennes propres si elle n'est pas Viennoise ou vient d'une région non germanique, ou bien lui demander d'utiliser un des livres de cuisine qui sont produits en quantité au tournant du siècle. II en résulte que les repas sont une combinaison de l'inspiration de la cuisinière et des exigences d'un train de maison bourgeois. Si ce dernier est aisé, la cuisinière doit être capable de préparer des dîners et des réceptions où il ne sied pas qu'elle serve de la nourriture "paysanne".

Ce qui est aujourd'hui dénommé cuisine viennoise n'a pas été formulé ainsi avant 1918 , ou bien très rarement ${ }^{217}$. De même que la nostalgie pour le multiculturalisme de la monarchie des Habsbourg, ses traditionsculinaires ont été érigées en lieu de mémoire immatériel ${ }^{218}$. Vienne a certes influencé tout l'Empire ; elle a exporté le Kaffeehaus et nombre d'autres habitudes culturelles, mais sa cuisine était elle-même un conglomérat d'influences. Le transfert s'est donc opéré dans les deux directions. L'inspiration viennoise est patente derrière la prolifération des hôtels et restaurants baptisés avec des noms "impériaux" : cette pratique s'est étendue jusqu'en Galicie, Bucovine et Bosnie-Herzégovine. La civilisation du Kaffeehaus règne d'un bout à l'autre de l'Empire ${ }^{219}$.

217

Wolf Neuber, Die k.u.k. Wiener Küche. Rezepte aus der guten alten Zeit, Munich, Mary Hahn, 1987.

218

Moritz Csáky, Georg Christian Lack (dir.), Kulinarik und Kultur: Speisen als kulturelle Codes in Zentraleuropa, Vienne, Böhlau, 2014.

Richard Kurdiovsky, « Das Kaffeehaus in der Doppelmonarchie. Zwischen Wiener Typus und nationalem Ausdruck ", in András F. Balogh (hrsg.), Mehrsprachigkeit in Zentraleuropa: zur Geschichte einer literarischen und kulturellen Chance, Vienne, Praesens-Verlag, 2012, p. 189-206. 
La première indication qui révèle ces échanges dans la diversité réside dans les dénominations des ingrédients et des recettes ; dans leur version autrichienne elles sont désormais considérées comme "typiques" de l'ancienne monarchie car elles diffèrent de celles en usage en Allemagne. Elles constituent donc un élément dans la construction de l'identité autrichienne mais elles sont en réalité un héritage d'autres langues que l'allemand $^{220}$. Les mots et leurs implications culinaires ont voyagé dans l'ensemble de l'Empire. Une illustration de cela peut être vue dans l'occupation de la Bosnie et de l'Herzégovine à partir de 1878: le vocabulaire autrichien se répandit dans tous les domaines dont la gastronomie avec l'arrivée des hôteliers d'Autriche dont la plupart étaient en fait des Tchèques et des Croates, et de Hongrie (surtout des Croates et des Serbes). Ils introduisirent les goûts austro-hongrois qui se combinèrent alors avec les spécialités turco-balkaniques.

La gastronomie n'a pas échappé aux querelles nationalistes, comme le démontre un exemple de Trieste. Le périodique slovène Slovenka (La Slovène) avait une rubrique dédiée au " pur slovène ». Se présentant sous forme de série, il s'agit d'un dictionnaire où les auteurs expliquent l'étymologie des mots étrangers qui ont pénétré la langue slovène. Ils préconisent de les remplacer par des termes "originaux » qu'ils sont parfois obligés de créer de toutes pièces. Ainsi le melšpajz forgé à partir de l'autrichien Mehlspeise (desserts à base de farine) doit être rebaptisé testenina. Au sujet des italianismes, les makaroni doivent être substitués par les vlitki, supposés plus autochtones ${ }^{221}$. Dans le même ordre d'idées, des restaurateurs de Zagreb rédigeant leurs menus en les truffant de termes allemands et/ou hongrois sont dénoncés dans la presse pour leur absence de conscience nationale, de surcroît quand ces personnes ne sont pas considérées comme "purement " croates. Toutefois la langue dans laquelle les menus étaient écrits ne devient pas un sujet de contentieux avant les années 1900. Et même alors, le besoin de

220

Julia Danielczyk, Birgit Peter, "Die Transnationale des Geschmacks », in Moritz Csáky, Georg Christian Lack, Kulinarik und Kultur Speisen..., op. cit., p. 69-82.

221

Slovenka. Glasiloslovenskegazenstva [La Slovène. La voix de la femme slovène], $\mathrm{n}^{\circ} 6,13$ mars, $\mathrm{n}^{\circ} 7,27$ mars, $\mathrm{n}^{\circ} 8,10$ avril et $\mathrm{n}^{\circ} 10,24$ avril 1897. 
standardisation émane du légalisme des autorités municipales et n'est donc pas à interpréter strictement en termes de nationalisme ${ }^{222}$. Or les hôteliers étaient effectivement réticents et lents à introduire une terminologie croate encore imprécise: ce sont finalement les termes allemands, compris de tous, qui s'imposèrent. En outre un grand nombre d'hôteliers et de restaurateurs étaient d'origine germanique. On adopta en définitive la solution des menus bilingues.

On peut donc considérer les cuisinières viennoises comme des médiatrices entre les nationalités puisque leur capacité à s'adapter n'avait que peu de rapport avec leur identification avec un groupe linguistique. Selon Konrad Köstlin : " in der Wiener Küche tanzt aber ein ganzes Ensemble ${ }^{223}$ ». La variété des plats que l'on pouvait trouver à la carte des cafés et restaurants de Vienne est l'expression de ce Volkstopf, avec toutefois des nuances suivant le niveau social du lieu. Depuis le Beis/ du coin de la rue à l'hôtel Sacher, du Heurigen de Grinzing à la pâtisserie Demel, le client trouvait plus ou moins les mêmes choses, ce qui aurait été impensable à Paris par exemple. Les noms et la terminologie circulaient de même, parfois avec des variantes importantes d'un livre de cuisine à un autre. Les transferts linguistiques étaient à l'œuvre sur tout le territoire austro-hongrois : italiens (frittata, risotto, fagioli), hongrois (gulyás, palacsinta). Les mots slaves, yiddish et turcs étaient plus ou moins correctement traduits avant d'être incorporés dans la gastronomie viennoise où ils étaient fondus de telle sorte que leurs sources d'inspiration originelles finissaient par être oubliées avant d'être quelquefois redécouvertes.

Même à la cour de Vienne, des plats simples constituaient l'ordinaire. François-Joseph était réputé pour apprécier le Tafelspitz (pot-au-feu) et nombre de ses dévoués sujets l'imitaient le dimanche ainsi que le raconte Joseph Roth dans La marche de Radetzky. Pour sa préparation, il est probable que la gouvernante, Fräulein Hirschwitz, n'avait point besoin de

222

Ines Sabotić, « Le menu à Zagreb au tournant du $X I X^{e}$ au $X X^{e}$ siècle : dans quelle langue mange-t-on ? », Balkanologie, n8, 2004, p. 55-70.

223

«Tout un orchestre fait danser la cuisine viennoise », Konrad Köstlin, «Die Wiener Küche. Ein alleinstellungsmerkmal avant la lettre », in Moritz Csáky, Georg Christian Lack (hrsg.), Kulinarik und Kultur Speisen..., op. cit., p. 121. 
livre de cuisine, tant ce plat était partie intégrante de la tradition culinaire autrichienne. Le goût manifesté par l'empereur pour le Tafelspitz était proverbial mais aussi ses visites à la pâtisserie Zauner lors de ses séjours à Bad Ischl, à tel point que le confiseur obtint l'honneur de fournir la cour (k.u.k. Hofzuckerbäcker), une distinction jusque-là réservée à Demel à Vienne et à quelques autres.

II est permis de penser que le culte voué aux gâteaux, à la crème et aux confiseries de toutes sortes est lié à la tradition baroque et à la piété catholique spécifiquement autrichienne (pietas austriaca) avec son décorum, ses illusions de stucs et ses formes théâtrales qui ont accompagné la Contre-Réforme. L'héritage des influences turques et italiennes a fait le reste en donnant aux populations des pays habsbourgeois le bec sucré. Le résultat est une parfaite combinaison entre la cuisine des gens modestes (crêpes, beignets) et la confiserie la plus raffinée. L'étalage des gâteaux (Torten) chez Demel à Vienne n'a rien à envier à l'abondante décoration des églises voisines.

Les livres ont été un élément primordial dans le processus de l'invention de la tradition de la cuisine austro-hongroise. Nombre d'entre eux ont été publiés au tournant du siècle, dans la plupart des cas par des femmes désireuses d'éduquer leurs cuisinières et les jeunes épouses. Les cuisinières de Bohême-Moravie avaient à leur disposition la bible de la cuisine tchèque de Magdalena Dobromila Rettigová, régulièrement rééditée et même traduite en allemand ${ }^{224}$ ! Elle s'adressait à la femme bourgeoise en phase d'émancipation et fait le lien avec le réveil national tchèque, mais elle parlait aussi à de plus modestes lectrices vivant dans des petites villes et à la campagne en dépit d'un niveau de langage soutenu. L'auteur a sélectionné environ 600 recettes issues de la heimatliche Tradition et affirme qu'elles sont tchèques. La plupart des cuisinières tchèques venant à Vienne en portaient un exemplaire dans leur valise. En effet, autour de 1880, 46,8 \% des femmes travaillant comme domestiques dans les foyers viennois venaient de Bohême ou de Moravie. Le premier livre faisant état de cette influence parut en 1911 sous la plume

224

Domácíkuchařka, anebpojednaní o masitých a postníchpokrmech pro dcerkyčeské a moravské. cité in Elisabeth Fendl, Jana Nosková, "Die böhmische Küche », in Heinke M. Kalinke, Klaus Roth, Tobias Weger (eds), Esskultur und kulturelle Identität, Berlin, De Gruyter, 2010, p. 105-136. 
de Johann Michael Heitz. Son Wiener Bürger-Küche donne une liste de recettes qualifiées de "böhmisch».

L'un des plus fameux livres de cuisine, avec ses 80 éditions jusqu'à la fin $\mathrm{du} \mathrm{xx}^{\mathrm{e}}$ siècle et traduit dans seize langues a été publié en 1858. Son auteure, Katharina Prato, est née à Graz en 1818. Elle développa ses dons de cuisinière en soignant son époux qui souffrait de l'estomac ${ }^{225}$. Les pages de Die Süddeutsche Küche s'adressaient à des novices en cuisine et aux femmes au foyer. Ce fut un succès immédiat ce qui encouragea l'auteure à ajouter de nouvelles recettes et à réviser continuellement son texte. Sa petite-fille, Viktorine von Leitmeier, poursuivit les mises à jour jusque dans l'entre-deux-guerres, introduisant de nouvelles recettes à ce qui s'appelait dorénavant Die große Prato. D'autres bestsellers en la matière ne manquèrent pas, tel que l'ouvrage de Marie von Rokitansky, ÖsterreichischeKüche, publié en 1897, ou bien celui d'Olga et Adolf Hess, Wiener Küche, paru en 1913. Ces livres furent également traduits dans diverses langues de la monarchie mais leurs recettes se retrouvèrent plus ou moins adaptées dans de nombreux livres originaux édités à travers tout l'Empire puisque certaines étaient issues de ses territoires, ce qui prouve une fois de plus le transfert entre centre et périphéries.

L'anthropologue Konrad Köstlin juge trompeur le terme de « cuisine viennoise » car selon lui, la cuisine de Vienne à la fin de l'ère impériale n'existe pas: il s'agirait en fait d'une construction a posteriori ${ }^{226}$. Or la définition de la soi-disant Wiener Küche commença à se former durant les dernières décennies $d u X x^{e}$ siècle à partir d'une compilation de plusieurs sources d'influences accumulées depuis le $x v l^{e}$ siècle. La dimension de l'Empire conditionna la variété des importations depuis ses périphéries proches et lointaines, un phénomène qui dura jusqu'à sa chute. Le cadre impérial, la cour, les autres centres du pouvoir (Espagne, l'Italie de la Lombardie à la Sicile, les Pays-Bas) créèrent des zones de contact entre

225

Rudolf Trefzer, «Das kulinarische Erbe der Donaumonarchie - Katharina Prato ", in Norbert Schreiber, Lojze Wieser (hrsg.), Wie schmeckt Europa?, Klagenfurt, Wieser Verlag, 2009, p. 163.

226

"Le résultat d'une auto-narration de la fin du XIX ${ }^{\mathrm{e}}$ siècle », Elisabeth Fendl, Jana Nosková, « Die böhmische Küche », op. cit., p. 105. 
territoires permettant un transfert continuel d'influences tel qu'il est impossible de déterminer l'origine de l'emblématique Wiener Schnitzel. Par son caractère de creuset des diverses populations de l'Empire, Vienne en vint à concentrer aussi une large part de l'héritage culinaire européen. La capitale recueillit ces influences grâce à la mobilité des habitants de la monarchie, au premier rang desquels les chefs et les cuisiniers. On pourrait donc pousser le raisonnement et considérer qu'il n'y pas plus de cuisine viennoise que d'identité autrichienne. II n'est ainsi pas très étonnant de voir que dans l'entre-deux-guerres, puis au lendemain de 1945, l'héritage culinaire a constitué un élément important de la construction de cette identité problématique. Dans les livres récemment publiés sur cette question, plusieurs chapitres sont consacrés à la cuisine viennoise ${ }^{227}$. Un lieu de mémoire a été créé ainsi avec l'aide des nombreuses marques anciennement k.u.k. Hoflieferanten. Elles jouent toujours un rôle dans la renommée internationale de Vienne, donnant même naissance à de nouveaux produits supposés procurer aux clients une idée de l'Empire. Le même phénomène est manifeste dans les pays de l'ancien bloc socialiste ayant appartenu auparavant à la monarchie et où des cafés "viennois » ont ouvert ou rouvert; leurs salles sont décorées de photographies « fin de siècle » qui montrent une image idéale de la ville avant 1918, ainsi que des portraits de François-Joseph et de « Sissi ».

Roman Sandgruber, "Österreichische Nationalspeisen. Mythos und Realität », in Hans J. Teuteberg, Gerard Neunamm, Alois Wierlacher (hrsg.), Essen und kulturelle Identität: europäische Perspektiven, Berlin, AkademieVerlag, 1997, p. 187-191. 